\title{
Bulk Crystallization Behavior of Poly(e-caprolactone) with a Wide Range of Molecular Weight
}

\author{
Wen-Chung Ou-YANG, ${ }^{\dagger}$ Lain-Jong LI,${ }^{*}$ Hsin-Lung Chen, ${ }^{* *}$ and Jenn Chiu HwanG*** \\ Department of Chemical Engineering, National Kaohsiung Institute of Technology, \\ Kaohsiumg, Taiwan, Republic of China \\ * Department of Chemistry, National Taiwan University, \\ Taipei, Taiwan, Republic of China \\ ** Department of Chemical Engineering, National Tsing Hua University, \\ Hsin-Chu, Taiwan, Republic of China \\ *** Department of Chemical Engineering, Yuan Ze Institute of Technology, \\ Nei-Li, Taoyuan, Taiwan, Republic of China
}

(Received February 17, 1997)

\begin{abstract}
Bulk crystallization kinetics and crystallizability of poly( $\varepsilon$-caprolactone) (PCL) with number average molecular weight $\left(M_{n}\right)$ ranging from 1900 to 64700 were investigated. Most PCL samples crystallized rapidly at 30 and $40^{\circ} \mathrm{C}$, such that the crystallization started before the equilibration of DSC signal. As a consequence, the relative crystallinity required for Avrami analysis could not be calculated accurately from the recorded DSC exotherms. An alternative method is proposed to evaluate the Avrami crystallization rate constant from the peak time of the isothermal crystallization exotherm. The calculated crystallization rate constants of PCL displayed a maximum in variation with molecular weight (MW). Such behavior was attributed to interplay between the MW effects on the thermodynamic driving force and the segmental mobility associated with crystallization. A kinetic formula proposed by Hoffman was used to obtain the crystal surface free energy product $\left(\sigma \sigma_{\mathrm{e}}\right)$. In contrast to the conventional Lauritzen-Hoffman analysis based on crystallization rates measured at different crystallization temperatures $\left(T_{\mathrm{c}}\right)$ for a given $\mathrm{MW}$, the present analysis was based on rates measured for different $\mathrm{MW}$ at a given $T_{\mathrm{c}}$. MW variation of degree of crystallinity also displayed a maximum rather than a monotonic drop. Crystallizability was suggested to be controlled by the presence of uncrystallizable short chains and the entanglements in the polymer. The fraction of the uncrystallizable short chains played the main role in controlling the crystallinity for the low MW samples, while entanglement density was the principal factor for the high MW samples. KEY WORDS Poly( $\varepsilon$-caprolactone) / Crystallization / Molecular Weight / Crystallization Kinetics / Crystallinity /
\end{abstract}

Poly( $\varepsilon$-caprolactone) (PCL) is a semicrystalline biodegradable polymer with a glass transition temperature $\left(T_{\mathrm{g}}\right)$ of $c a .-60^{\circ} \mathrm{C}$ and observed melting point around $55^{\circ} \mathrm{C}$. Extensive research of PCL and its blends has been reported. ${ }^{1-9)}$ In a prior study, ${ }^{10}$ we prepared PCL with a wide range of molecular weight $(\mathrm{MW})\left(M_{n}\right.$ ranging from 1900 to 64700) via fractionation by either precipitating PCL/chloroform solutions into different amounts of methanol or adding methanol into PCL/tetrahydrofuran (THF) solutions. The samples thus obtained were used to investigate the MW effects on the spherulite growth rate, equilibrium melting point $\left(T_{\mathrm{m}}^{0}\right)$, nucleation density, and morphology of PCL. The variation of spherulite growth rate with MW was found to exhibit a maximum rather than conventional monotonic drop. A growth kinetics analysis was also devised to extract the crystal surface free energy based on a MW-growth rate relationship proposed by Hoffman. This analysis was conducted using MW as the variable but crystallization temperature $\left(T_{\mathrm{c}}\right)$ as a fixed parameter.

In addition to spherulite growth, bulk crystallization behavior such as bulk crystallization kinetics and crystallizability is also a very important aspect of crystalline polymers. The present study extends the previous investigation to discuss the influence of $\mathrm{MW}$ on bulk crystallization kinetics and crystallizability of PCL.

\footnotetext{
† To whom correspondence should be addressed.
}

\section{EXPERIMENTAL}

The fractionation procedures for obtaining PCL with a wide range of $\mathrm{MW}$ is described in the previous publication. ${ }^{10}$ The absolute molecular weights of all the samples were determined by a Kratos Model Spectraflow 400 Gel Permeation Chromatography (GPC) equipped with Shodex KF-801, KF-802, KF-803, and KE-804 columns and Viscotek Model-100 differential refractometer/ viscometer detectors. The average molecular weights $\left(M_{n}, M_{w}\right.$, and $\left.M_{z}\right)$ were obtained through the universal calibration curve using the Viscotek Unical GPC software ver. 4.01. PCL samples with $M_{n}$ ranging from 1900 to 64700 were used in the present study. The $M_{n}$ and $M_{w}$ of some selective samples are listed in Table I. The samples used here had similar polydispersity indices around 1.2 to 1.3 . Thus molecular weight distribution was not considered as a principal variable here.

Bulk crystallization behavior was investigated with a Perkin-Elmer DSC-7 differential scanning calorimeter (DSC). For the crystallization kinetics study, the sample was first annealed at $80^{\circ} \mathrm{C}$ for $3 \mathrm{~min}$ to erase previous thermal history followed by rapid cooling at $c a .120^{\circ} \mathrm{C}$ $\mathrm{min}^{-1}$ to the desired $T_{\mathrm{c}}\left(30\right.$ or $\left.40^{\circ} \mathrm{C}\right)$ where the isothermal crystallization exotherm was recorded. For crystallizability evaluation, the degree of crystallinity for PCL isothermally crystallized at 25 to $45^{\circ} \mathrm{C}$ for $11 \mathrm{~h}$ was calculated from the enthalpy of melting $\left(\Delta h_{\mathrm{f}}\right)$. The degree of crystallinity was calculated by $\Delta h_{\mathrm{f}} / \Delta h_{\mathrm{f}}^{0}$ with $\Delta h_{\mathrm{f}}^{0}=$ $117 \mathrm{~J} \mathrm{~g}^{-1}$, the enthalpy of melting of $100 \%$ crystalline 
Table I. $\quad M_{n}$ and $M_{w}$ of PCL samples in this study

\begin{tabular}{crr}
\hline Sample ID & $M_{n}$ & $M_{w}$ \\
\hline 1 & 2700 & 3200 \\
2 & 2800 & 3700 \\
3 & 4400 & 5700 \\
4 & 6300 & 7300 \\
5 & 6700 & 8500 \\
6 & 19600 & 26300 \\
7 & 22400 & 27600 \\
8 & 29800 & 36200 \\
9 & 44300 & 55400 \\
10 & 44700 & 51700 \\
11 & 64700 & 77600 \\
\hline
\end{tabular}

PCL. ${ }^{11}$ The DSC scanning rate was $20^{\circ} \mathrm{C} \mathrm{min}^{-1}$.

\section{RESULTS AND DISCUSSION}

The influence of MW on the bulk crystallization kinetics of PCL at 30 and $40^{\circ} \mathrm{C}$ was investigated. Figure 1 displays the representative isothermal crystallization exotherms of three samples. For samples of $M_{n}=2800$ and 22400 the crystallizations were so rapid that they started before the equilibration of DSC signals and hence the initial portions of the exotherms were masked by the equilibration signal. This was observed for most PCL samples under study. Since the whole crystallization exotherm could not be detected intactly, error would be introduced if the observed exotherm was used to calculate the relative crystallinity for performing the conventional Avrami analysis. Although the initial portion of the exotherm was masked by the equilibration signal, the peak time $\left(t_{\mathrm{p}}\right)$ of the exotherm was detected without perturbation. $t_{\mathrm{p}}$ of the exotherm is related to the crystallization rate. Shorter $t_{\mathrm{p}}$ would imply a faster crystallization rate. Since $t_{\mathrm{p}}$ can be measured accurately, if a quantitative relationship between crystallization rate constant and $t_{\mathrm{p}}$ is available, the rate constant can be calculated from $t_{\mathrm{p}}$. Such a quantitative relationship may be formulated easily by considering the Avrami equation:

$$
x_{\mathrm{c}}(t)=1-\exp \left(-k_{n} t^{n}\right)
$$

where $x_{\mathrm{c}}(t)$ is relative crystallinity, $n$ is the Avrami exponent depending on the nucleation mechanism and growth geometry, $k_{n}$ is the crystallization rate constant. It is noted that the rate constant is expressed with a subscript $n, k_{n}$, to indicate that the unit of the rate constant is $[\mathrm{s}]^{-n}$ dependent on the value of $n$. If the rate constant is written as $k$, the rate constant is given by $k=k_{n}^{1 / n}$ and has the unit of $[\mathrm{s}]^{-1}$. The signal recorded by DSC is proportional to the time derivative of $x_{\mathrm{c}}(t)$, $\mathrm{d} x_{\mathrm{c}}(t) / \mathrm{d} t$; therefore, $t_{\mathrm{p}}$ corresponds to the maximum of $\mathrm{d} x_{\mathrm{c}}(t) / \mathrm{d} t$. Then we write,

$$
\frac{\mathrm{d}}{\mathrm{d} t}\left(\frac{\mathrm{d} x_{\mathrm{c}}(t)}{\mathrm{d} t}\right)=0
$$

at $t=t_{\mathrm{p}}$. Substituting eq 1 for $x_{\mathrm{c}}(t)$ in the above equation, it can be shown that

$$
k_{n}=\frac{(n-1)}{n t_{\mathrm{p}}^{n}}
$$

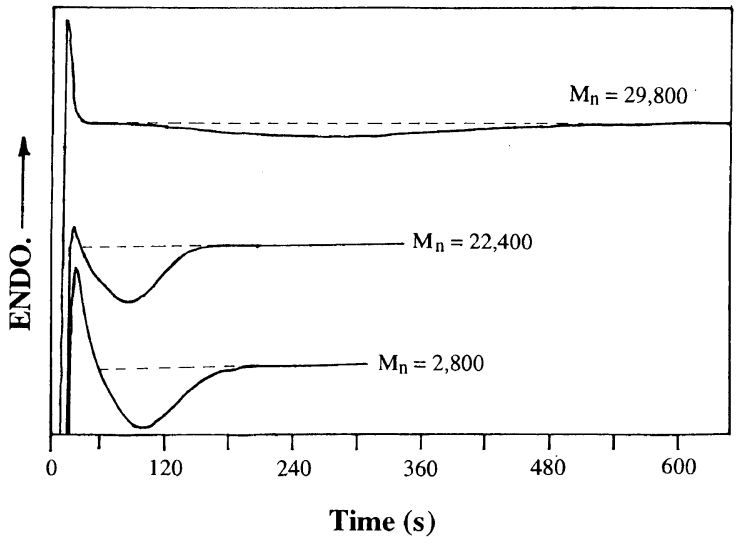

Figure 1. DSC isothermal crystallization exotherms of PCL with $M_{n}=2800,22400$, and 29800 . The crystallization temperature was $40^{\circ} \mathrm{C}$. The initial portion of the crystallization exotherms was masked by the equilibration signals for $M_{n}=2800$ and 22400 .

or

$$
k=\left[\frac{(n-1)}{n}\right]^{1 / n} t_{\mathbf{p}}^{-1}
$$

The rate constants can be calculated by eq 2 and 3 once $t_{\mathrm{p}}$ and $n$ are known. Equations 2 and 3 prescribe that a larger value of $t_{\mathrm{p}}$ corresponds to a smaller $k$. Since Avrami equation does not consider the contribution of secondary crystallization to $x_{\mathrm{c}}(t)$, the rate constants calculated by eq 2 and 3 are only associated with the primary crystallization. Therefore, when the observed $t_{\mathrm{p}}$ is used to calculate the rate constants by eq 2 and 3 , it has tacitly assumed that the occurrence of secondary crystallization did not shift the location of $t_{\mathrm{p}}$ associated with the primary crystallization exotherm. This is probably a sound assumption, since secondary crystallization normally accounts for less than $35 \%$ of the total crystallinity and only becomes important at the late stage of crystallization where primary crystallization is close to the end. ${ }^{12}$ Calculations of rate constants by eq 2 and 3 also assume that the equilibration of the DSC signal does not perturb the actual position of $t_{\mathrm{p}}$.

In addition to $t_{\mathrm{p}}$, the value of $n$ is also necessary for calculating the rate constants by eq 2 and 3 . The value of $n$ can be evaluated from the slope of the Avrami plot $\left(\ln \left[-\ln \left(1-x_{\mathrm{c}}(t)\right)\right] v s . \ln t\right)$ for some samples whose crystallization exotherms can be detected without overlapping with the equilibration signal. $n=3$ is the typical value which signifies a thermodynamically controlled crystallization with instantaneous nucleation and spherulitic growth geometry. The Avrami plots of two PCL samples whose crystallization exotherms were recorded intactly are shown in Figure 2. The slopes of the plots are about $2.5 \pm 0.5$. The value of $n=2.5$ would imply a diffusion-controlled crystallization with homogeneous nucleation and spherical growth geometry. However, crystallization kinetics of PCL at the temperatures under investigation (i.e., 30 and $40^{\circ} \mathrm{C}$ ) is controlled by the thermodynamic driving force of crystallization rather than mobility of the molecules. This was evidence by the observation that crystallization rate decreased with increasing temperature (or decreasing degree of supercooling) within this temperature range. ${ }^{10}$ Since the uncertainty associated with the determination of $n$ was 0.5 , 


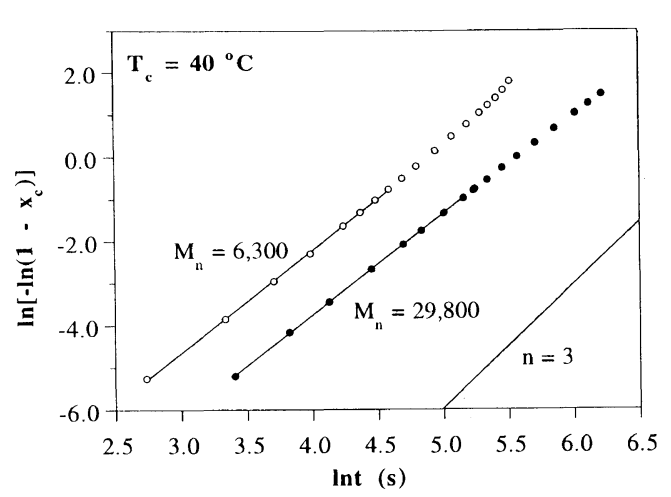

Figure 2. Avrami plots of PCL samples whose crystallization exotherms could be recorded intactly without overlapping with the equilibration signals.

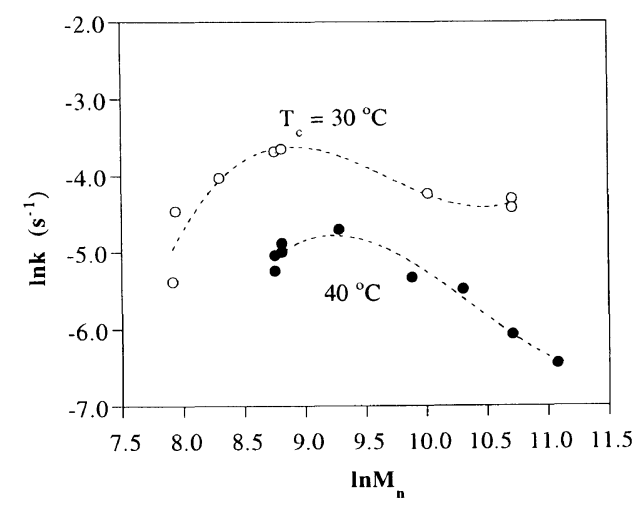

Figure 3. Variation of crystallization rate with MW for PCL. The variation displayed a maximum rather than a monotonic drop.

we adopted the value of 3 as the Avrami exponent to calculate the rate constants by eq 3 . The value of $n=3$ appears more reasonable because it stands for a thermodynamically-controlled crystallization with spherulitic growth geometry. The spherulitic morphology has been confirmed for all PCL samples in the prior study. ${ }^{10}$

The calculated crystallization rate constants for $T_{\mathrm{c}}=30$ and $40^{\circ} \mathrm{C}$ are plotted against $\ln M_{n}$ in Figure 3 . The calculated rate constants for the two samples in Figure 2 were $\ln k\left(\mathrm{~s}^{-1}\right)=-5.24$ and -5.48 for $M_{n}=6300$ and 29800 respectively, which agrees quite well with the rate constants, -5.56 and -5.01 , obtained from the intercepts of the Avrami plots. It can be seen in Figure 3 that the variation of rate constants with MW displayed a maximum rather than a monotonic drop. The existence of such a maximum was attributed to the dependence of crystallization rate on $T_{\mathrm{m}}^{0}$ and segmental mobility. Increasing MW exerted two opposing effects on the crystallization rate of a polymer; one to reduce the segmental mobility which would result in retardation of crystallization rate, while the other, to increase $T_{\mathrm{m}}^{0}$ and hence the degree of supercooling which would promote the crystallization rate. Interplay between these two will determine the observed crystallization rate and may generate a maximum observed in Figure 3. At low $\mathrm{MW}$, the effect of $T_{\mathrm{m}}^{0}$ increase was more pronounced than reduction in segmental mobility, and the crystallization rate increased with $\mathrm{MW}$; the opposite was true at high MW.

The dependence of crystallization rate on MW can be described by the following equation ${ }^{13}$

$$
k=\left(\frac{k_{0}}{\xi}\right) \exp \left(-\frac{Q_{\mathrm{D}}^{*}}{R T_{\mathrm{c}}}\right) \exp \left[-\frac{\alpha b_{0} \sigma \sigma_{\mathrm{e}} T_{\mathrm{m}}^{0}(\xi)}{k_{\mathrm{B}} \Delta h_{\mathrm{f}}^{0} \Gamma_{\mathrm{c}}\left[T_{\mathrm{m}}^{0}(\xi)-T_{\mathrm{c}}\right]}\right]
$$

where $k_{0}$ is a constant, $\xi$ is the average number of units in a polymer chain, $Q_{\mathrm{D}}^{*}$ is the activation energy for the reptation process, $\sigma$ and $\sigma_{\mathrm{e}}$ are the side and fold surface free energy, respectively, $b_{0}$ is the monomolecular thickness, $\Delta h_{\mathrm{f}}^{0}$ is the bulk enthalpy of melting per unit volume of crystal, and $\alpha$ is a constant depending on the growth regime. If the fold structure of the crystal surface does not vary with MW (although tie chains may increase with $\mathrm{MW}$, their contribution to the crystal surface is considered to be much smaller than chain folds; therefore, the crystal surface is assumed to be unaffected by $\mathrm{MW}), \sigma_{\mathrm{e}}$ can be assumed independent of MW. The logarithmic form of eq 4 can thus be rearranged to give

$$
\ln k+\ln \xi=\ln W\left(T_{\mathrm{c}}\right)-\psi\left(T_{\mathrm{c}}\right) \frac{T_{\mathrm{m}}^{\mathrm{o}}(\xi)}{T_{\mathrm{m}}^{0}(\xi)-T_{\mathrm{c}}}
$$

where

$$
\ln W\left(T_{\mathrm{c}}\right)=\ln k_{0}-\frac{Q_{\mathrm{D}}^{*}}{R T_{\mathrm{c}}}
$$

and

$$
\psi\left(T_{\mathrm{c}}\right)=\frac{\alpha b_{0} \sigma \sigma_{\mathrm{e}}}{k_{\mathrm{B}} \Delta h_{\mathrm{f}}^{0} T_{\mathrm{c}}}
$$

Equation 5 shows that a plot of $\ln k+\ln \xi v s . T_{\mathrm{m}}^{0}(\xi) /$ $\left[T_{\mathrm{m}}^{0}(\xi)-T_{\mathrm{c}}\right]$ for a given $T_{\mathrm{c}}$ yields a straight line with the slope of $-\psi\left(T_{\mathrm{c}}\right)$. Once $\psi\left(T_{\mathrm{c}}\right)$ is obtained the surface free energy product $\sigma \sigma_{\mathrm{e}}$ can be calculated. This appears to be an alternative method to obtain the crystal surface free energy based on the crystallization kinetic study, while $\sigma \sigma_{\mathrm{e}}$ has typically been obtained by studying the crystallization rate at various $T_{\mathrm{c}} \mathrm{s}$ for a fixed $\mathrm{MW}$.

The above analysis was performed for $T_{\mathrm{c}}=30$ and $40^{\circ} \mathrm{C}$ using the calculated crystallization rate constants. $\xi$ was calculated by $\xi=M_{n}{ }^{\prime} M_{u}$ with $M_{u}$ the MW of a unit. $M_{u}$ was taken as $114 \div 7 \mathrm{~g} \mathrm{~mol}^{-1}$ here because a caprolactone unit - $\left(\mathrm{CH}_{2}\right)_{5} \mathrm{COO}$ - has a molecular weight of 114 and is considered to contain seven units. $T_{\mathrm{m}}^{0}$ of PCL corresponding to different MW was extrapolated by a Hoffman-Weeks plot, previously. ${ }^{10}$ The dependence of $T_{\mathrm{m}}^{0}$ on $\mathrm{MW}$ was expressed as

$$
T_{\mathrm{m}}^{0}(\xi)=353.0 \frac{(118.9+\xi)}{(132.3+\xi)}
$$

where the unit of $T_{\mathrm{m}}^{0}(\xi)$ described by the above equation is $K$. Substituting eq 8 for $T_{\mathrm{m}}^{0}(\xi)$ in eq 5 , the plot of $\ln k+\ln \xi$ vs. $T_{\mathrm{m}}^{0}(\xi) /\left[T_{\mathrm{m}}^{0}(\xi)-T_{\mathrm{c}}\right]$ was constructed as shown in Figure 4. It appears that the slopes or $-\psi\left(T_{\mathrm{c}}\right)$ of the two linear lines are not the same. The value of $-\psi\left(T_{\mathrm{c}}\right)$ for $T_{\mathrm{c}}=30^{\circ} \mathrm{C}$ is -1.23 which is 2.2 times that for $T_{\mathrm{c}}=40^{\circ} \mathrm{C}$. This is in agreement with the observation using spherulite growth rate for the analysis. ${ }^{10}$ Equation 7 shows that $\psi\left(T_{\mathrm{c}}\right)$ is proportional to $\alpha$ whose value is determined by the growth regime; $\alpha=4$ for regimes I and III while $\alpha=2$ for regime II. The difference in $\psi\left(T_{\mathrm{c}}\right)$ between the two $T_{\mathrm{c}} \mathrm{s}$ is hence due to different 


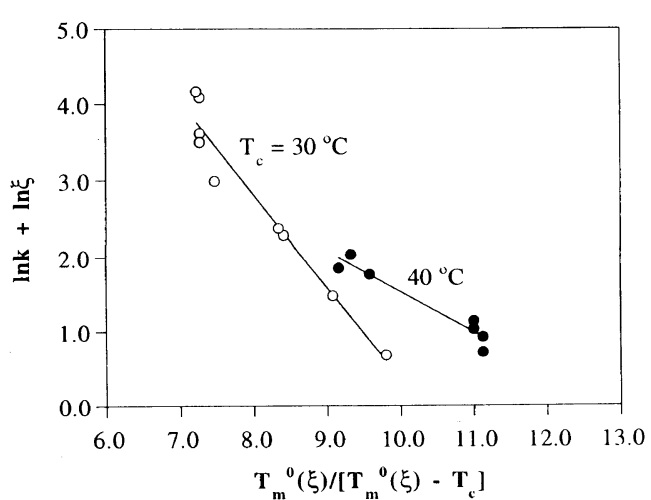

Figure 4. $\ln k+\ln \xi v s . T_{\mathrm{m}}^{0}(\xi) /\left[T_{\mathrm{m}}^{0}(\xi)-T_{\mathrm{c}}\right]$ plot for PCL. The slopes of the linear line for $T_{\mathrm{c}}=30^{\circ} \mathrm{C}$ was about two times that for $T_{\mathrm{c}}=40^{\circ} \mathrm{C}$.

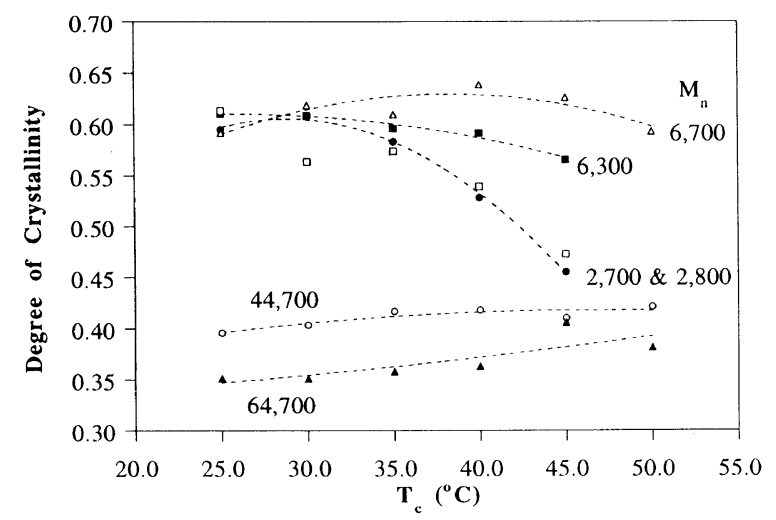

Figure 5. Degree of crystallinity of PCL crystallized at different $T_{\mathrm{c}} \mathrm{s}$. The crystallinity decreased with increasing $T_{\mathrm{c}}$ for the samples with $M_{n}<10000$, while the opposite was observed for the two samples with $M_{n}=44700$ and 64700 .

regimes the crystal growth is associated. Crystal growth of PCL took place in regime II at $40^{\circ} \mathrm{C}$ but in regime III at $30^{\circ} \mathrm{C}$; consequently, $\psi\left(30^{\circ} \mathrm{C}\right)$ was about two times $\psi\left(40^{\circ} \mathrm{C}\right)$. Using the values of $b_{0}=4.13 \AA$ and $\Delta h_{\mathrm{f}}^{0}=$ $1.48 \times 10^{9} \mathrm{erg} \mathrm{cm}^{-3}, 6 \sigma \sigma_{\mathrm{e}}$ was calculated as $460 \mathrm{erg}^{2} \mathrm{~cm}^{-4}$ and $432 \mathrm{erg}^{2} \mathrm{~cm}^{-4} \mathrm{using} \psi\left(30^{\circ} \mathrm{C}\right)$ and $\psi\left(40^{\circ} \mathrm{C}\right)$, respectively. These values were about $21 \%$ lower than obtained from the crystallization rate at various $T_{\mathrm{c}} \mathrm{s}$ (conventional Lauritzen-Hoffman analysis). ${ }^{1}$ Determination of $\sigma \sigma_{\mathrm{e}}$ using the conventional method needs to assume the values of the activation energy of mobility $\left(U^{*}\right)$ and temperature at which the segmental transport is prohibited $\left(T_{\infty}=T_{\mathrm{g}}-C\right) . U^{*}=1500 \mathrm{cal} \mathrm{mol}^{-1}$ and $T_{\infty}=$ $T_{\mathrm{g}}-30$ were adopted for PCL in the previous study. ${ }^{1}$ The method proposed here does not require such assumptions because all the parameters (such as $\xi$ and $\left.T_{\mathrm{m}}^{0}(\xi)\right)$ can be obtained experimentally.

The effect of MW on the crystallizability of PCL was investigated. The degree of crystallinity normally decreased with increasing $\mathrm{MW},{ }^{14,15}$ probably due to the higher entanglement density associated with the polymer of higher MW polymer. Figure 5 displays the degree of crystallinity calculated from the enthalpy of melting for PCL crystallized at different $T_{\mathrm{c}} \mathrm{s}$ for $11 \mathrm{~h}$. The crystallinity is seen to depend on $\mathrm{MW}$ and $T_{\mathrm{c}}$. For the four samples with $M_{n}$ less than 10000 , the crystallinity decreased with increasing $T_{\mathrm{c}}$ and the drop was more obvious for lower MW samples. The opposite was observed

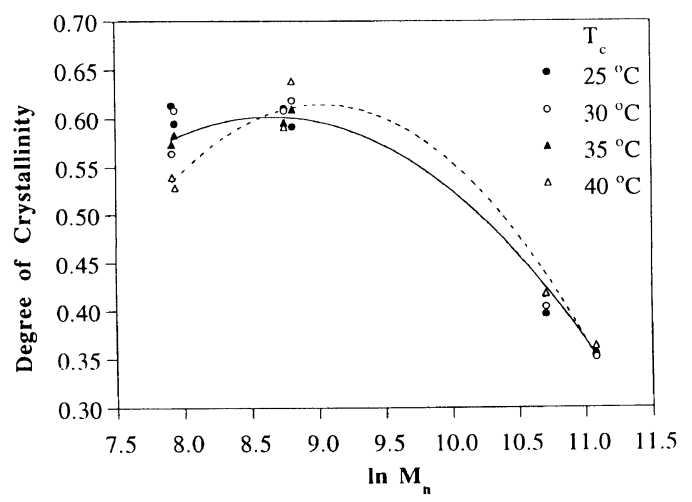

Figure 6. Variation of crystallinity with MW for PCL. The variation displayed a maximum rather than monotonic drop.

for the samples with high $M_{n}$ of 44700 and 64700 . Figure 6 plots the degree of crystallinity against $\ln M_{n}$. Just like the crystallization rate, the variation of crystallinity with MW also displayed a maximum. For the lower MW polymer, there existed a portion of short chains whose $T_{\mathrm{m}}^{0}$ were close or even lower than $T_{\mathrm{c}}$; as a result, they were not able to crystallize at $T_{\mathrm{c}}$. As $T_{\mathrm{c}}$ increased, the fraction of these uncrystallizable short chains increased, and hence the crystallinity dropped with increasing $T_{\mathrm{c}}$. For higher $\mathrm{MW}$ samples, the amounts of short chains whose $T_{\mathrm{m}}^{0}$ were close or lower than $T_{\mathrm{c}}$ was low, and crystallizability was predominantly controlled by the entanglement density. As $T_{\mathrm{c}}$ increased, the polymer chains had higher mobility and hence they could release the restraints imposed by entanglements more easily. As a consequence, the crystallinity increased with $T_{\mathrm{c}}$. The above interpretation suggests that for a given type of polymer with various $\mathrm{MW}$, the crystallizability is controlled by the fraction of short chains with low $T_{\mathrm{m}}^{0}$ and the entanglement density. For the low MW polymer, the crystallizability is predominantly controlled by the fraction of short chains such that the crystallinity increases with increasing MW but decreases with increasing $T_{\mathrm{c}}$. The crystallizability is predominantly controlled by the entanglement density for the high MW sample, the crystallinity thus decreases with increasing MW but increases with $T_{\mathrm{c}}$.

\section{CONCLUSIONS}

The bulk crystallization kinetics and crystallizability of PCL with a wide range of MW have been investigated. A method was devised to obtain the Avrami crystallization rate constants from the peak time of the isothermal crystallization exotherm. This method should be useful as the crystallization rate is so fast such that the entire crystallization exotherm cannot be recorded by DSC. Just like the spherulite growth rate, the variation of crystallization rate constants with MW displayed a maximum for PCL. This observation was attributable to interplay between the MW effects on $T_{\mathrm{m}}^{0}$ and segmental mobility associated with crystallization.

A new method is proposed to obtain the crystal surface free energy product $\left(\sigma \sigma_{\mathrm{e}}\right)$. This analysis is based on the rates measured for different $\mathrm{MW}$ at a given $T_{\mathrm{c}}$. Determination of $\sigma \sigma_{\mathrm{e}}$ using the conventional Lauritzen- 
Hoffman analysis needs to assume the values of the activation energy of mobility $\left(U^{*}\right)$ and the temperature at which segmental transport is prohibited $\left(T_{\infty}=T_{\mathrm{g}}-\right.$ $C)$. The method proposed here does not require such assumptions as all the parameters used can be obtained experimentally.

MW variation of degree of crystallinity also displayed a maximum in contrast to a conventional monotonic drop. The presence of uncrystallizable short chains and entanglement density were suggested to control the crystallizability of the polymer. For low MW samples, the presence of uncrystallizable short chains was more important and hence the crystallinity decreased with decreasing MW and increasing $T_{\mathrm{c}}$. For high MW samples, the entanglement density played the major role such that the crystallinity decreased with increasing MW and decreasing $T_{\mathrm{c}}$.

\section{REFERENCES}

1. P. J. Philips, G. J. Rensch, and K. D. Taylor, J. Polym. Sci., Part B, Polym. Phys., 25, 1725 (1987).

2. J. M. Jonza and R. S. Porter, Macromolecules, 19, 1946 (1986).

3. S. Nojima, H. Tsutsui, M. Urushihara, W. Kosaka, N. Kato, and T. Ahida, Polym. J., 8, 451 (1986).

4. K. R. Chynoweth and Z. H. Stachurski, Polymer, 27, 1912 (1986).

5. J. M. Vion, R. J'erome, P. Teyssie', M. Aubin, and R. E. Prud'homme, Mocromolecules, 19, 1828 (1986).

6. L. Goulet and R. E. Prud'homme, J. Polym. Sci., Part B, Polym. Phys., 28, 2329 (1990).

7. S. H. Goh and M. K. Neo, Eur. Polym. J., 9, 927 (1991).

8. Y. W. Cheung and R. S. Stein, Macromolecules, 27, 2512 (1994).

9. A. A. C. M. Oudhuis, H. J. Thiewes, P. F. van Hutten, and G. ten Brinke, Polymer, 35, 3936 (1994).

10. H.-L. Chen, L.-J. Li, W.-C. Ou-Yang, J. C. Hwang, and W.-Y. Wong, Macromolecules, 30, 1718 (1997).

11. B. Lebedev and A. Levstropov, Makromol. Chem., 185, 1235 (1984).

12. B. Hsiao, J. Polym. Sci., Polym. Phys. Ed., 31, 237 (1993).

13. J. D. Hoffman, Polymer, 23, 656 (1982).

14. L. Mandelkern, A. L. Allou, and M. Gopalan, J. Phys. Chem., 72, 309 (1968).

15. M. Glotin and L. Mandelkern, Colloid Polym. Sci., 260, 182 (1982). 